\title{
High-Contrast Interference in a Thermal Cloud of Atoms
}

\author{
D. E. Miller, J. R. Anglin, J. R. Abo-Shaeer, K. Xu, J. K. Chin, and W. Ketterle* \\ Department of Physics, MIT-Harvard Center for Ultracold Atoms, \\ and Research Laboratory of Electronics, MIT, Cambridge, MA 02139
}

(Dated: September 3, 2018)

\begin{abstract}
The coherence properties of a gas of bosonic atoms above the BEC transition temperature were studied. Bragg diffraction was used to create two spatially separated wave packets, which interfere during expansion. Given sufficient expansion time, high fringe contrast could be observed in a cloud of arbitrary temperature. Fringe visibility greater than $90 \%$ was observed, which decreased with increasing temperature, in agreement with a simple model. When the sample was "filtered" in momentum space using long, velocity-selective Bragg pulses, the contrast was significantly enhanced in contrast to predictions.
\end{abstract}

PACS numbers: PACS 03.75.Fi, 34.20.Cf, 32.80.Pj, 33.80.Ps

Images of interfering atomic clouds are widely considered a hallmark of Bose-condensed systems and a signature of long-range correlations [1]. A thermal atomic cloud is often regarded as an incoherent source, with a coherence length too short to obtain high-contrast interference patterns when two clouds are overlapped. Here we show that ballistic expansion can increase the coherence length such that "BEC type" interference can be observed in a thermal cloud. Currently, there is considerable interest in characterizing the coherence properties of non-condensed systems including ultracold fermions 2], fermion pairs [3, 4, 5] and ultra-cold molecules [6, 7, 8]. In this paper we show that an interferometric autocorrelation technique, previously only applied to condensates [9, 10], can be used to study the coherence properties of samples at finite temperature.

We studied the first-order spatial coherence of a trapped thermal cloud of atoms. After release from the trap, the atom cloud expanded ballistically, and Bragg diffraction was used to create an identical copy of the initial cloud displaced by a distance $d$. Therefore, our study was analogous to Young's double-slit experiment [1]. We investigate the conditions under which two such overlapping clouds produce a high-contrast interference pattern. Our result is that for sufficiently long expansion times, there will always be high contrast, but the required time-of-flight becomes longer at higher temperatures.

The experiment used a magnetically trapped thermal cloud of $\sim 5 \times 10^{7}$ sodium atoms, prepared in a manner similar to our previous work [12]. Atoms in the $\left|F=1, m_{F}=-1\right\rangle$ state were loaded from a MOT into a magnetic trap, where they were further cooled by radio frequency (RF) evaporation. The RF evaporation was stopped before the critical temperature for Bose-Einstein condensation (BEC) $T_{c}$ was reached, yielding a thermal cloud at a controlled temperature. Shortly after being re-

\footnotetext{
*Website: cua.mit.edu/ketterle_group
}
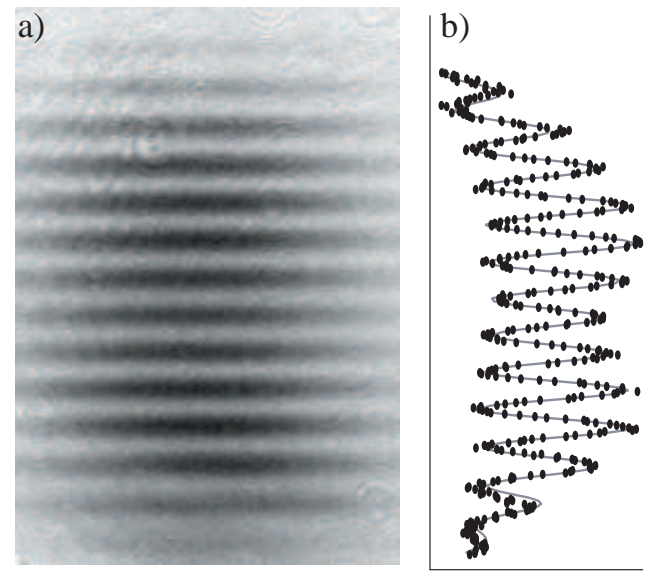

c)

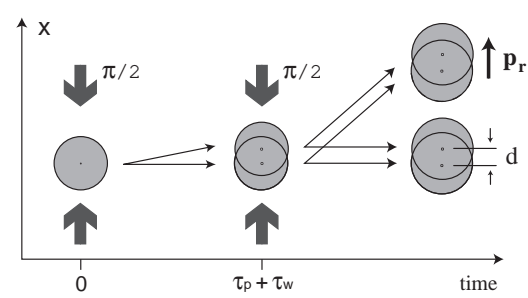

FIG. 1: Interference of spatially separated thermal clouds: (a) An absorption image, taken after $48 \mathrm{~ms}$ time of flight, shows fringes with a spatial period of $\lambda_{f}=340 \mu \mathrm{m}$. (b) A cross section of the optical density (black circles) taken through the center of the image was fit (grey line) to extract the fringe contrast. (c) Schematic depiction of the Bragg pulse sequence. The first $\pi / 2$ pulse at $t=0$ created a superposition of stationary and moving clouds. At $t=\tau_{p}+\tau_{w}$ the clouds had separated by distance $d$, and a second pulse created a superposition of cloud pairs; one pair moving with recoil momentum $\mathbf{p}_{r}$, the other stationary. Each pair developed an interference pattern.

leased from the trap (2 ms), the cloud was exposed to two successive Bragg pulses, separated by wait time $\tau_{w}$. The effect of the Bragg beams [13, 14] was to couple two momentum states $\left|\hbar \mathbf{k}_{0}\right\rangle$ and $\left|\hbar\left(\mathbf{k}_{0}+\mathbf{k}_{r}\right)\right\rangle$, via a two photon transition, where $\mathbf{k}_{r}=\left(\mathbf{k}_{1}-\mathbf{k}_{2}\right)$ and $\mathbf{k}_{1}, \mathbf{k}_{2}$ are the wave vectors of the two Bragg beams. The coupling induced 


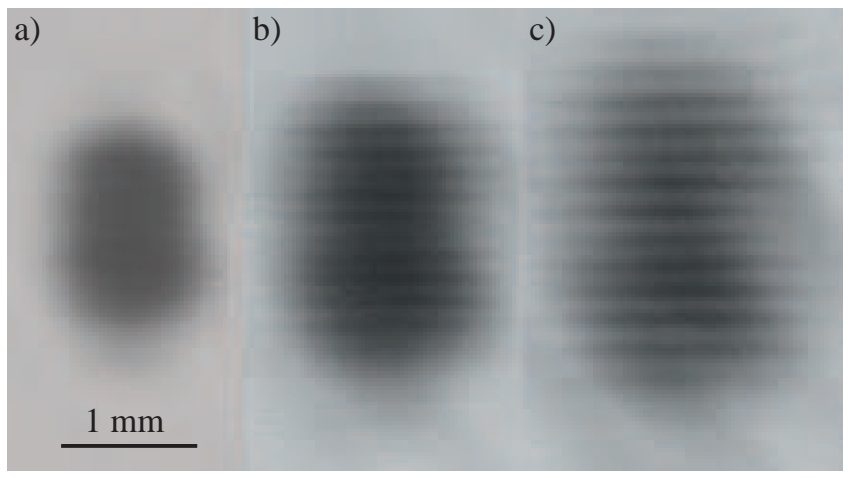

FIG. 2: Emerging contrast during ballistic expansion. The coherence length $\ell_{c}$ grew larger than the initial separation $\mathrm{d}=2 \mu \mathrm{m}$ as the cloud expanded for (a) $14 \mathrm{~ms}$, (b) $20 \mathrm{~ms}$, (c) $25 \mathrm{~ms}$. (see Eq. 4)

Rabi oscillations and the pulse area was experimentally chosen to correspond to $\pi / 2$ (i.e., an atom originally in a well defined momentum state was taken to an equal superposition of the two states). During the wait time $\tau_{w}$ the two states accrued different phases before a second $\pi / 2$ pulse mixed the states again. Considering only two momentum states, this is equivalent to Ramsey spectroscopy [15].

One can regard a thermal cloud as an ensemble of atoms, each having its initial amplitude spread over a range of momentum states centered about zero, with r.m.s. width $h / \lambda_{T}$, where $\lambda_{T}=h / \sqrt{m k_{B} T}$ is the thermal de Broglie wavelength and $m$ the atomic mass. The relative detuning of $\delta \nu=45 \mathrm{KHz}$ between the Bragg beams and pulse duration $\tau_{p}=10 \mu s$ were chosen to couple all initial momentum components to those centered at $\mathbf{k}_{r}$. During $\tau_{w}$ the relative phase accumulated between coupled states is proportional to $k_{0}$, and the momentum distribution shows a sinusoidal modulation (Ramsey fringes) after the Bragg sequence. In long timeof-flight (tof), the spatial density simply mimics this momentum distribution. Interference fringes were observed at a spacing $\lambda_{f}=h t_{\text {tof }} / m d$, where

$$
d=v_{r}\left(\tau_{w}+4 / \pi \tau_{p}\right)
$$

is the cloud separation discussed in the equivalent picture of two overlapping clouds. $v_{r}$ is the two-photon recoil velocity. The factor of $4 / \pi$ emerges from a simple Rabi oscillation model, and includes the extra phase accumulated while the Bragg beams effect a $\pi / 2$ pulse. The sum of single particle interference patterns results in a density along the $\mathrm{x}$-direction with reduced contrast $\mathrm{C}$ :

$$
n(x)=f(x)\left[1+C \sin \left(\frac{2 \pi}{\lambda_{f}} x+\phi\right)\right] .
$$

where $f(x)$ is an envelope function.

To find the expected contrast we consider the $k$-space distribution of noninteracting bosons in a harmonic trap

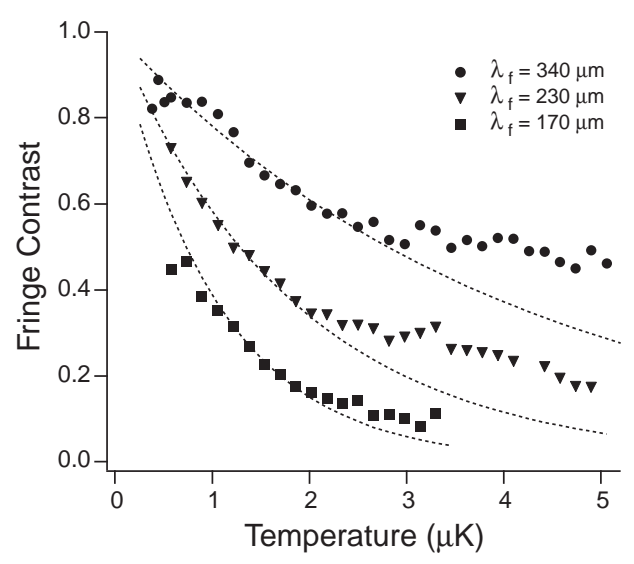

FIG. 3: Contrast vs. temperature for $10 \mu \mathrm{s}$ Bragg pulses. Different fringe spacings $\lambda_{f}$ were realized by varying the wait time $\tau_{w}$ between the two Bragg pulses. The time-of-flight was kept fixed at $48 \mathrm{~ms}$. At lower temperatures the observed contrast agreed well with theory (dashed lines) given by Eq. 3 At higher temperature, however, the contrast was higher than predicted, owing to the velocity selectivity of the Bragg pulses. The BEC transition temperature was at $T_{c}=0.6 \mu \mathrm{K}$.

assuming the high temperature (Maxwell-Boltzmann) limit. This may be written exactly as an ensemble of single-particle Gaussian states, incoherently averaged over their positions. Thus, it is appropriate to regard the thermal cloud as a collection of wave packets of gaussian width $\lambda_{T}$, distributed in space according to MaxwellBoltzmann statistics.

The incoherent sum over all particles results in contrast

$$
C=\exp \left(-\frac{2 \pi^{2} R_{T}^{2}}{\lambda_{f}^{2}}\right)=\exp \left(-\frac{d^{2}}{2 \ell_{c}^{2}}\right)
$$

where $R_{T}=\sqrt{k_{B} T / m \omega^{2}}$ is the thermal size of a cloud in a harmonic trap of frequency $\omega$ and where we have defined the coherence length as

$$
\ell_{c}=\frac{\lambda_{T}}{2 \pi} \frac{\text { size in tof }}{\text { size in trap }}
$$

Eq. 3 gives two different, but equivalent criteria for the loss of contrast. While the interference pattern of a single-particle quantum state (as well as that of a pure condensate) should always show perfect contrast, the incoherent sum over a thermal cloud washes out the fringe visibility. Contrast is lost when the single particle interference pattern is smeared out over an initial size $R_{T}$ that is larger than the fringe spacing $\lambda_{f}$. However, because the fringe spacing grows as the cloud expands, contrast will always emerge with enough time of flight. Alternatively, Eq. 3 states that interference is lost when the separation $d$ of the two sources exceeds the coherence length $\ell_{c}$. Here it is important to note, that the coherence length increases with time-of-flight (Eq. 4). The coherence length 


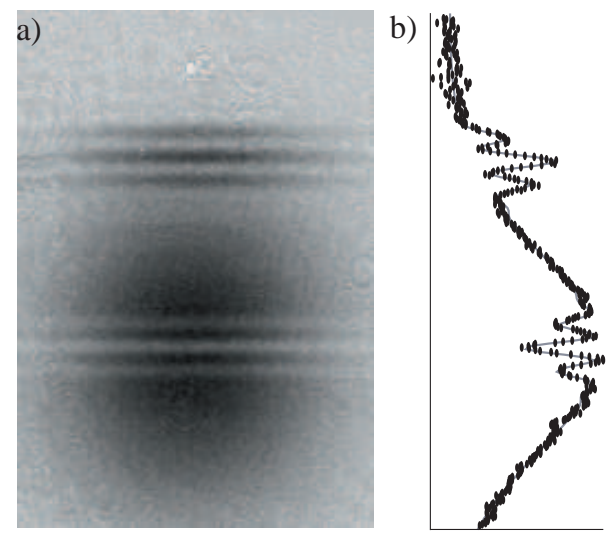

FIG. 4: Velocity selective Bragg diffraction: (a) Absorption image of atoms subject to $40 \mu \mathrm{s}$ Bragg pulses. The fringe spacing was $\lambda_{f}=210 \mu \mathrm{m}$ after $48 \mathrm{~ms}$ time-of-flight. These longer Bragg pulses addressed only a subset of the momentum distribution. (b) Cross section and fit (black circles and grey line respectively).

is inversely proportional to the local momentum spread which decreases in ballistic expansion as atoms with different velocities separate from each other. For very long expansion times, the coherence length becomes arbitrarily large resulting in high-contrast interference (Fig. 2). This can also be understood by the conservation of local phase space density during ballistic expansion, where the decrease in density is accompanied by a decrease in momentum spread.

We repeated the experiment over a range of temperatures for several values of the cloud separation $d$. The temperature was controlled by varying the final value of the RF evaporation. Each temperature was calibrated by measuring the size of a cloud in expansion without pulsing on the Bragg beams. The temperature calibration was consistent with the observed onset of BEC at the calculated temperature of $T_{c}=0.6 \mu K$. The Bragg beams were detuned $30 \mathrm{GHz}$ from the atomic transition and heating was demonstrated to be negligible by observing the effects of the light with the two-photon detuning $\delta \nu$ set far from the Bragg resonance. Absorption images of our samples were taken after $48 \mathrm{~ms}$ time-of-flight (Fig. 1a). A cross section of the atomic density was fit to Eq. 2 to determine the contrast. Deviation of the pulse area from $\pi / 2$ reduces the number of atoms in the out-coupled cloud, however it does not reduce the contrast assuming the two pulses are equal.

Fig. 3a shows the measured fringe contrast of the outcoupled cloud as a function of the cloud temperature for three different fringe spacings $\lambda_{f}$. The data is compared to the contrast expected from Eq. 3 (dashed line) with no free parameters. At low temperature this equation provides an accurate description of the observed fringe visibility. At higher temperatures, however, the observed contrast is consistently greater than expected. While

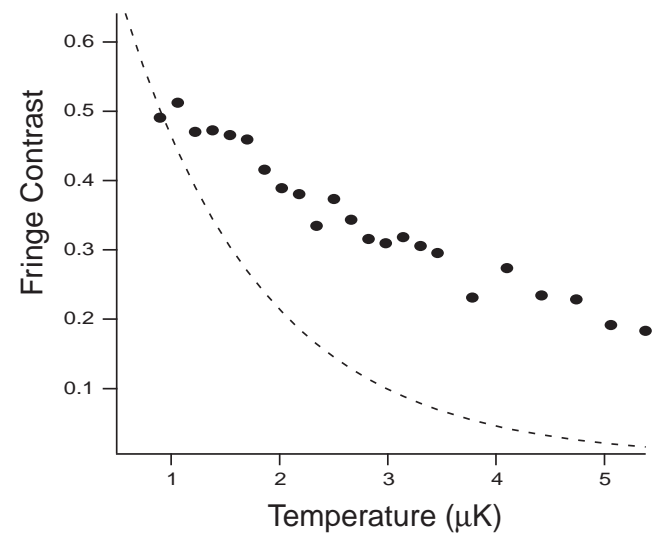

FIG. 5: Measured contrast for $\tau_{p}=30 \mu s$ velocity selective Bragg pulses. The contrast was significantly higher than that predicted by Eq. 3 (dashed line). The fringe spacing was $\lambda_{f}=170 \mu \mathrm{m}$ after $48 \mathrm{~ms}$ expansion time.

Bose-Einstein statistics can considerably enhance contrast at temperatures even above $T_{c}$ [16, 17], our experiment does not achieve the high densities necessary to make this effect pronounced. One possible explanation for the discrepancy in Fig. 5 is that our model fails when the Bragg diffraction becomes velocity selective, i.e., when the Doppler width of the atoms exceeds the Fourier width of the Bragg pulses.

In order to further investigate this effect, we repeated the experiment with longer, more velocity-selective pulses. The absorption images showed that these pulses addressed a narrow range of atomic velocities (Fig. 4). In this case the fit routine was modified to account for the (Gaussian) background of atoms unaffected by the Bragg pulses. While the fraction of out-coupled atoms decreased with increasing temperature, the interference was still clearly visible. In Fig. 5 the measured contrast deviates substantially from theory (dashed line). While velocity selectivity clearly plays a role, the mechanism for this enhanced contrast is not apparent. We have shown that it is only the initial cloud size $R_{T}$ that determines the contrast for a given $\lambda_{f}$ (Eq. (3). Since the Bragg pulses are not spatially selective, we do not expect their details

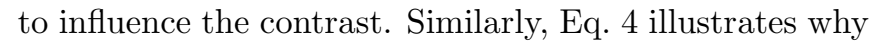
velocity selection does not increase the coherence length in time-of-flight: the narrowed momentum distribution implies a larger effective de Broglie wavelength which is exactly cancelled by the reduced expansion in time-offlight. Therefore, the enhanced contrast in Fig. 5 cannot be described by the single-particle free expansion of a thermal gas. We suspect that particle interactions play a role.

In conclusion, we have shown how the coherence length of a trapped gas is modified during ballistic expansion. This allows for the observation of high-contrast interference in a sample at any temperature, and is not limited to condensates. For the technique employed here, 
the only advantage of a BEC is its larger initial coherence length, which is equal to the size of the condensate 1, 9, 16]. However, it is the ability of two independent condensates to interfere that sets this state of matter apart [18]. Two independent thermal clouds would not interfere. The self-interference technique characterized here can be used to study the coherence properties of other novel quantum degenerate systems. One example are molecular clouds created by sweeping an external magnetic field across a Feshbach resonance. While the rapid decay of such a sample precluded thermalization, an interferometric method has already been used to demonstrate coherence in this system [19].

The authors thank D. E. Pritchard for critical reading of the manuscript, M. W. Zwierlein for contributions to the data analysis and W. Setiawan for experimental assistance. This research is supported by NSF, ONR, ARO and NASA.

[1] M. R. Andrews, C. G. Townsend, H.-J. Miesner, D. S. Durfee, D. M. Kurn, and W. Ketterle, Science 275, 637 (1997).

[2] A. Cho, Science 301, 750 (2003).

[3] C. A. Regal, M. Greiner, and D. S. Jin, Phys. Rev. Lett. 92, 040403 (2004).

[4] M. W. Zwierlein, C. A. Stan, C. H. Schunck, S. M. F. Raupach, A. J. Kerman, and W. Ketterle, Phys. Rev. Lett. 92, 120403 (2004).

[5] C. Chin, M. Bartenstein, A. Altmeyer, S. Riedl,
S. Jochim, J. H. Denschlag, and R. Grimm, Science 305, 1128 (2004).

[6] J. Herbig, T. Kraemer, M. Mark, T. Weber, C. Chin, H.-C. Ngerl, and R. Grimm, Science 301, 1510 (2003).

[7] S. Dürr, T. Volz, A. Marte, and G. Rempe, Phys. Rev. Lett. 92, 020406 (2004).

[8] K. Xu, T. Mukaiyama, J. R. Abo-Shaeer, J. K. Chin, D. E. Miller, and W. Ketterle, Phys. Rev. Lett. 91, 210402 (2003).

[9] E. Hagley, L. Deng, M. Kozuma, M. Trippenbach, Y. Band, M. Edwards, M. Doery, P. Julienne, K. Helmerson, S. L. Rolston, et al., Phys. Rev. Lett. 83, 3115 (1999).

[10] J. E. Simsarian, J. Denschlag, M. Edwards, C. W. Clark, L. Deng, E. W. Hagley, K. Helmerson, S. L. Rolston, , and W. D. Phillips, Phys. Rev. Lett. 85, 2040 (2000).

[11] O. Carnal and J. Mlynek, Phys. Rev. Lett. 66, 2689 (1991).

[12] J. R. Abo-Shaeer, C. Raman, J. M. Vogels, and W. Ketterle, Science 292, 476 (2001).

[13] P. J. Martin, B. G. Oldaker, A. H. Miklich, and D. E. Pritchard, Phys. Rev. Lett. 60, 515 (1988).

[14] D. M. Giltner, R. W. McGowan, and S. A. Lee, Phys. Rev. A 52, 3966 (1995).

[15] N. F. Ramsey, Rev. Mod. Phys. 62, 541 (1990).

[16] I. Bloch, T. Hänsch, and T. Esslinger, Nature 403, 166 (2000).

[17] M. Naraschewski and R. J. Glauber, Phys. Rev. A 59, 4595 (1999).

[18] M. Naraschewski, H. Wallis, A. Schenzle, J. I. Cirac, and P. Zoller, Phys. Rev. A 54, 2185 (1996).

[19] J. R. Abo-Shaeer, D. E. Miller, J. K. Chin, K. Xu, T. Mukaiyama, and W. Ketterle, Phys. Rev. Lett. 94, 040405 (2005). 